\title{
Comparison of HR-pQCT- and microCT-based finite element models for the estimation of the mechanical properties of the calcaneus trabecular bone
}

\author{
J. Alsayednoor ${ }^{1} \cdot$ L. Metcalf ${ }^{2} \cdot$ J. Rochester ${ }^{3} \cdot$ E. Dall'Ara ${ }^{4}$ E. McCloskey ${ }^{2,4} \cdot$ D. Lacroix $^{1}$
}

Received: 16 June 2017 / Accepted: 2 July 2018 / Published online: 10 July 2018

(c) The Author(s) 2018

\begin{abstract}
The calcaneus bone is formed of extensive trabecular bone and is therefore well suited to be used as an example of loaded bone to establish the ability of combining microfinite element (microFE) technique with high-resolution peripheral quantitative computed tomography (HR-pQCT) in determining its mechanical properties. HR-pQCT is increasingly used as a tool for in vivo bone clinical research, but its use has been limited to the distal radius and tibia. The goal of this study was to determine the applicability of HR-pQCT-derived microFE models of the calcaneus trabecular bone with $82 \mu \mathrm{m}$ voxel size with reference to higher-resolution microCT-based models taken as gold standard. By comparing the outputs of microFE models generated from both HR-pQCT and microCT images of the trabecular bone of five calcaneus cadaveric specimens, it was found that the HR-pQCT-based models predicted mechanical properties for fracture load, total reaction force and von Mises stress are considerably different from microCT-based counterparts by 33, 64 and 70\%, respectively. Also, the morphological analysis showed a comprehensive geometrical difference between HR-pQCT-based microFE models and their microCT-based equivalents. The results of the HR-pQCT-based models were found to have strong dependency on the threshold value chosen to binarise the images prior to finite element modelling. In addition, it was found that the voxel size has a strong impact on accuracy of imaged-based microFE models compared to other factors such as the presence of soft tissue and image scanning integration time. Therefore, although HR-pQCT has shown to be useful to predict overall structural and biomechanical changes, it is limited in providing local accurate biomechanical properties of trabecular bone and therefore should be used with caution when assessing bone remodelling through local changes of trabecular bone apposition and resorption in disease treatment monitoring.
\end{abstract}

Keywords In vivo assessment · Trabecular microstructure · Finite element model · HR-pQCT · Trabecular segmentation · Calcaneus

\section{Introduction}

D. Lacroix

D.Lacroix@sheffield.ac.uk

1 Department of Mechanical Engineering, INSIGNEO Institute for In Silico Medicine, University of Sheffield, Mappin Street, Sheffield S1 3JD, UK

2 Department of Oncology and Metabolism, MRC-Arthritis UK Centre for Integrated Research into Musculoskeletal Ageing (CIMA), University of Sheffield, Sheffield, UK

3 Academic Unit of Medical Education, Medical School, University of Sheffield, Sheffield, UK

4 Department of Oncology and Metabolism and INSIGNEO Institute for In Silico Medicine, University of Sheffield, Sheffield, UK
The calcaneus bone has important function within the human musculoskeletal system of bearing body weight during standing and motion. The large portion of this anatomical part consists of trabecular bone; age- and osteoporosis-related changes in calcaneal bone reflects bone strength at other skeletal sites, and the heel has been widely used to predict future fracture risk predominantly using measurements by quantitative ultrasound (McCloskey et al. 2015). The in vivo assessment of bone microstructure contributes to a better understanding of the pathophysiology of diseases such as osteoporosis as well as improving our understanding of the efficacy of therapeutic interventions (Farr and Khosla 2015; Fuller et al. 2015; Liebl et al. 2014; Paggiosi 
et al. 2014; Nishiyama and Shane 2013; Pialat et al. 2012). High-resolution peripheral quantitative computed tomography (HR-pQCT) is a non-invasive, low-radiation technique designed to measure bone microstructural properties at peripheral sites in vivo (Putman et al. 2014; Stein et al. 2014; Boutroy et al. 2008). Although the second-generation HRpQCT system (XtremeCT II) has provided improved image resolution (e.g. $61 \mu \mathrm{m}$ Manske et al. 2015 and $41 \mu \mathrm{m}$ EnnsBray et al. 2016), the first-generation XtremeCT I system ( $82 \mu \mathrm{m}$ voxel size) is still widely used for bone mechanical analysis purposes. Many recent HR-pQCT-based studies have followed a similar microFE modelling framework, which originated from the pioneering work of van Rietbergen and colleagues (van Rietbergen and Ito 2015; Van Rietbergen et al. 1995) who developed a methodology based on microCT scans of trabecular bone. The methodology was based on three main steps: (1) low-pass image filtration, (2) global single level threshold and (3) conversion of binarised image voxels into linear hexahedron elements. Since then, this modelling protocol has been revised and improved. For example, Laib and Ruegsegger (1999) applied a 3D Laplace-Hamming high-pass filter to effectively remove trabecular-edge noises from microCT images. In another study, Ulrich et al. (1998) studied the effect of mesh smoothing on microCT-based trabecular structure by using tetrahedral elements to improve the preservation of trabecular connectivity in comparison with hexahedral mesh. In another study, a volumetric topological analysis (Saha et al. 2010) was developed to classify the trabecular microstructure into plate/rod-like components, and the hexahedral elements were replaced by beam and shell elements according to their topological classification (Wang et al. 2015; Vanderoost et al. 2011).

However, it is still debated whether accurate finite element (FE) predictions can be obtained from HR-pQCT-imaged trabecular bone when the same procedures defined for microCT-based FE analysis are used. Bevill and Keaveny (2009) used a simple mesh resampling technique on human cadaveric trabecular microstructures (femoral neck, greater trochanter and vertebral body), obtained from microCT scan with $20 \mu \mathrm{m}$ voxel size. They concluded that scans with voxel size below $80 \mu \mathrm{m}$ can accurately predict bone strength. However, they did not account for the intrinsic reduction of signal-to-noise ratio of in vivo HR-pQCT images compared to a resampled microCT image. Depalle et al. (2013) also performed a similar investigation by considering L2 human cadaveric vertebrae, and they found that voxel size and degree of element formulation are important factors which directly impact on the accuracy of stress distribution within the trabecular microstructure in the FE model. In a more recent study, Christen et al. (2016) investigated the voxel size dependency of cadaveric distal radius compressive load by using microCT and generating microstructures using multiple downscaled images (from 50 to $150 \mu \mathrm{m}$ ) where they found small voxel size dependency at $61 \mu \mathrm{m}$ (average error in three orthogonal loading cases of $8.2 \%$ compared to $25-\mu \mathrm{m}$ gold-standard microCT-based model) and increased dependency at larger voxel sizes (average error of $16.7 \%$ for $82 \mu \mathrm{m}$ ). MacNeil and Boyd (2008) and Varga et al. (2011) investigated the accuracy of HR-pQCT-based microFE strength predictions (Pistoia et al. 2002) by conducting cadaveric distal radius uniaxial compressive test. In these studies, good agreement was observed between FE results and experimental data derived to calculate apparent bone strength. It should be noted that in distal radius sections the presence of dense cortical shell around the trabecular region acts as mechanical stabiliser in particular when the load is applied longitudinally. As a result, the cortical part of such a specimen bears more load than the trabecular bone. On the other hand, the cortical shell is more accurately converted to microFE compared to the trabecular region which has a wider range of greyscale distribution, if a single level threshold-based method is applied. Therefore, it is hard to understand whether the local predictions of HR-pQCT-based microFE models of distal radius or tibia are accurate in their trabecular core.

To investigate the HR-pQCT-based ( $82 \mu \mathrm{m}$ voxel size) FE prediction for human distal tibia by considering whole (cortex and trabecular part) and trabecular segment as well as cubic subvolumes of trabecular region, Liu et al. (2010) pioneered a direct morphological and FE mechanical comparative study where microCT-based models for each cadaveric specimen were considered as gold standard. In this study, despite the differences between HR-pQCT and microCT measurements, high correlations between the two modelling approaches were observed. To evaluate the trabecular bone behaviour more explicitly and using microCT-based models as a gold standard, Tjong et al. (2012) applied HR-pQCT imaging on cadaveric distal radius samples by considering multiple voxel sizes $(41,82$ and $123 \mu \mathrm{m})$ and found a strong correlation between HR-pQCT model voxel sizes and the accuracy of trabecular region morphometric parameters. In the latest study, Zhou et al. (2016) had examined the accuracy of HR-pQCT ( $82 \mu \mathrm{m}$ voxel size)based microFE predictions for human cadaveric distal radius and tibia by calculating and comparing mechanical properties (bone stiffness and yield strength) and morphological parameters against gold-standard microCT-based microFE and mechanical testing results. They reported a strong correlation between HR-pQCT- and microCT-based mechanical and morphological properties, where corrective adjustment was required to achieve the accurate values for HR-pQCTbased models in the light of their microCT counterparts. To the best of the authors' knowledge, there has not been any established systematic study on the morphological and mechanical properties of microFE models derived from calcaneum HR-pQCT scans. Therefore, by adopting the com- 
parative analysis approach from Liu et al. (2010) study, the aim of this study was to determine the influence of global thresholding technique of HR-pQCT on the morphological and biomechanical analyses of calcaneus trabecular bone when compared with microCT-based reconstructions and models.

\section{Materials and methods}

\subsection{Specimen preparation}

Five embalmed human cadaveric left feet were obtained from the University of Sheffield medical teaching unit where the study was approved by the 'Medical School Ethics and Research Committee'. The age of donors ranged from 85 to 101, all female. HR-pQCT scanning (XtremeCT I, SCANCO-V1.1c, Scanco Medical) of the specimens was performed in situ with two different integration times of 100 and $200 \mathrm{~ms}$. It should be noted that the scanning protocol with $100 \mathrm{~ms}$ integration time is the typical method used for distal tibia and distal radius. The other integration times could be chosen to decrease the scanning time and, therefore, decrease the probability of artefacts. All the other parameters were the same: $82 \mu \mathrm{m}$ isotropic voxel size, $60 \mathrm{kVp}$ voltage, $900 \mu \mathrm{A}$ current. Afterwards, a parallelepiped portion of the calcaneus $\left(\approx 18 \times 18 \times 33 \mathrm{~mm}^{3}\right)$ was extracted by means of a diamond bandsaw under constant water irrigation (300CP, Exakt, Germany) as shown in Fig. 1.

The extracted calcaneus specimens (fully dissected with removed soft tissues) were then scanned with a microCT system (SkyScan 1172, Bruker, Belgium) with the following parameters: $17.41 \mu \mathrm{m}$ isotropic voxel size, $2950 \mathrm{~ms}$ integration time, $100 \mathrm{kV}$ voltage, $100 \mu \mathrm{A}$ current, $180^{\circ}$ total rotation, $0.7^{\circ}$ rotation step, $2 \times$ averaging. The microCT images of parallelepiped portion of calcaneus and HR-pQCT

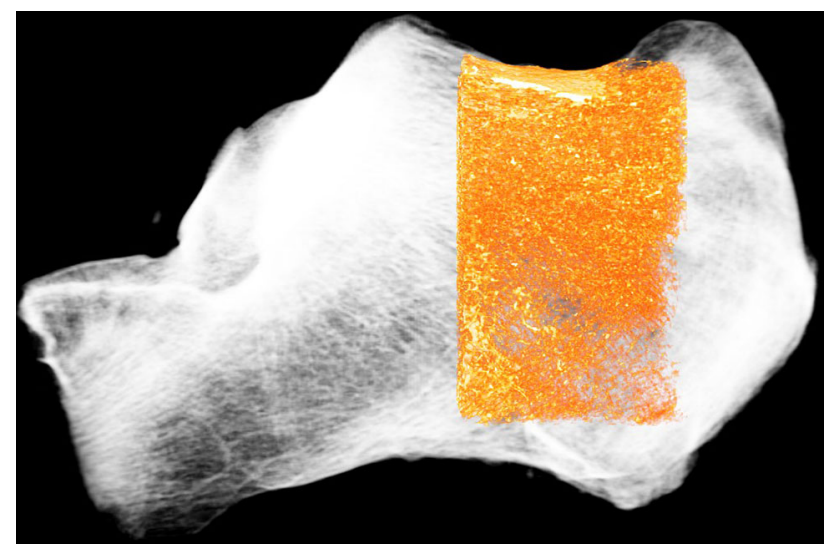

Fig. 1 An example of trabecular region (in orange) extracted from the dissected calcaneus (in white) for microCT imaging images of full feet (acquired with $100 \mathrm{~ms}$ integration time or $200 \mathrm{~ms}$ integration time, independent registrations) were rigidly registered using AMIRA 6.0.1 in order to select the same region of interest between the low- and high-resolution images.

\subsection{Thresholding}

To construct microCT-based trabecular models, $301 \times 301 \times$ 301 voxel cubic subvolumes were considered from the central region of the registered microCT data, where also the equivalent volumes were extracted from their corresponding HR-pQCT sets $(65 \times 65 \times 65$ voxels, one extra voxel size was considered on each side to fully capture the boundary geometry). Initially, the uint 16 microCT image slices were converted into uint 8 format and then filtered by applying low-pass Gaussian filter $(0.5,5)$. The gold standards for both the morphological parameters and the mechanical properties in this study were derived from the segmentations of the microCT images which were used to generate the microCT-based models. For a high-resolution microCT images, adopting a single level threshold method is an adequate technique to separate the pixels which represent bone from surrounding marrow/air (Ding et al. 1999). To select the right threshold, the BV/TV values were initially computed at different threshold ranges. Then, by differentiating the $\mathrm{BV} / \mathrm{TV}$ and threshold curve, the threshold value corresponding to the steepest curve gradient was chosen as the right input that was equal to 90 for all the specimens (Scanco Medical AG 1997). To study the effect of resampling method (similar to Bevill and Keaveny 2009), additional microstructures were also generated by resampling the original binarised microCT-based model using MATLAB-written resize function (k-wave 2015; That is, the model is downsampled by a factor of $17.41 / 82 \approx 0.2$ ) and a single value thresholding was applied to achieve BV/TV similar to the gold-standard microCT model (referred to as microC $\mathrm{T}_{\mathrm{Res}}$ ).

Due to lower resolution of the HR-pQCT images compared to the microCT ones, larger partial volume effect is expected and the calculation of the BV/TV is more affected by small changes in the single level threshold values. In particular, increasing the threshold value can lead to a significant trabecular network disconnection. To study this problem, two threshold values were considered to evaluate the HRpQCT-based structural and mechanical properties. For each specimen, the first threshold (TH1) was chosen to obtain $\mathrm{BV} / \mathrm{TV}$ values similar to those obtained from the goldstandard microCT images. However, with TH1 the microFE models generated from microCT or HR-pQCT were geometrically different (Fig. 2). Therefore, a second threshold value (TH2) was used, resulting in a more similar geometry to the microCT model. To achieve this, the fractal theory was employed (Alberich-Bayarri et al. 2010), which indi- 
Fig. 2 Illustrative representation of HR-pQCT-based modelling, using different threshold values. $\mathrm{TH}_{1}$ and $\mathrm{TH}_{2}$ represent the first and second threshold values, respectively. It should be noted that in general, there is not a linear trend between the threshold variation and the geometrical similarity between HR-pQCT- and microCT-based models (quantified using fractal dimension method)

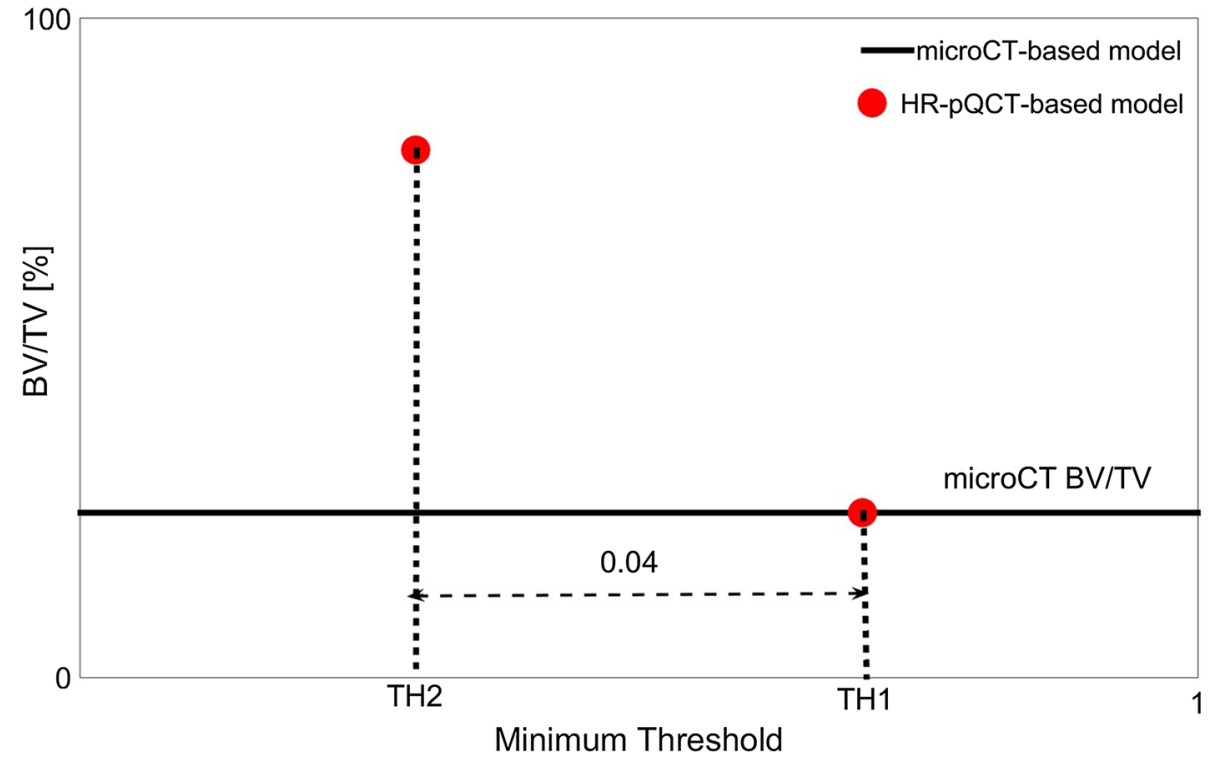

cates how the binarised trabecular structure is arranged at different dimensional scales and provides a systematic way to compare different microstructures. The fractal dimension curves were generated for each HR-pQCT image for different threshold ranges (Moisy 2008) and compared against the values obtained from the analyses of the corresponding microCT images. A threshold value TH2 equal to (TH1 0.04) minimised the differences in the microstructure between HRpQCT and microCT images. Nevertheless, TH2 leads to higher BV/TV for HR-pQCT images compared to those computed in microCT images (143 $\pm 79 \%$ increase; see Fig. 2). The HR-pQCT-based models generated using TH1 and TH2 are represented as HR-pQCT $\mathrm{TH}_{\mathrm{TH}}$ and $\mathrm{HR}-\mathrm{pQCT} \mathrm{T}_{\mathrm{TH} 2}$.

\subsection{FE computation}

The binarised voxels were converted to ABAQUS/Standard 6.13-1 cubic hexahedral elements (C3D8) where implicit solver was used for all simulations with linear elastic material property, $E=10 \mathrm{GPa}, v=0.33$ assigned to each element (Pistoia et al. 2002). It should be noted that the Pistoia rule was defined and validated for other human anatomical sites such as wrist where in this study it is assumed that this hypothesis behaves similarly for the calcaneus part. By considering three main loading directions, $0.007 \mathrm{~mm}$ uniaxial compression $(0.13 \%$ compressive strain) was applied on one end of all microstructures, while the opposite end was fully constrained similar to Boutroy et al. (2008) (see Fig. 3).

\subsection{Output processing}

von Mises stress (Stress $\left.\mathrm{vm}_{\mathrm{vm}}\right)$, total applied force $\left(F_{\text {tot }}\right)$ and estimated failure load $\left(F_{\text {frct }}\right)$ were computed from each FE simulation for each loading case (Vilayphiou et al. 2010,
2011; Wegrzyn et al. 2010). To predict $F_{\text {frct }}$, the Pistoia et al. (2002) criteria were used by assuming that bone failure would happen when at least $2 \%$ of the total volume of elements are loaded beyond $7000 \mu$ strain. At the end of each FE simulation, the elemental Strain $_{\text {eqv }}$ values and the total applied reaction force, $F_{\text {tot }}$, were extracted and imported to the MATLAB-based program to evaluate the $F_{\text {frct }}$.

\subsection{Microstructural classification}

In order to study the effect of geometrical anisotropy on the mechanical response, the degree of anisotropy DA and the main trabecular direction using MIL (mean interception length) technique were calculated (BoneJ plugin in ImageJ, NIH Image). Plateness/rodness, local thickness and heterogeneous arrangement of individual trabeculae within the microstructure are important morphological features which affect the mechanical response (Liu et al. 2008). Vasilic et al. (2009) established object classification criteria which provide a good indication of local trabeculae shape by computing eigenvalues of local structure tensor and then applying Eq. 1 to calculate the plate/rod ratio:

$C_{\mathrm{r}}=2\left[\frac{\lambda_{2}-\lambda_{1}}{\lambda_{3}-\lambda_{1}}\right]-1, \quad \lambda_{1}<\lambda_{2}<\lambda_{3}$

where $\lambda_{1}, \lambda_{2}$ and $\lambda_{3}$ represent the eigenvalues and $C_{\mathrm{r}}$ $\in(-1,1)$ where shifting towards -1 and 1 corresponds to more rod-like and plate-like geometries, respectively. For effective computation of local structure tensor, it is required to obtain the medial surface of trabecular microstructure by using so-called digital object skeletisation (or thinning) methodology. To achieve this, a 3D thinning algorithm was generated using MATLAB script based on original work of 


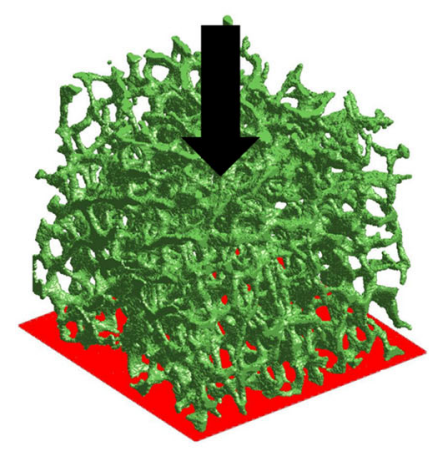

(a)

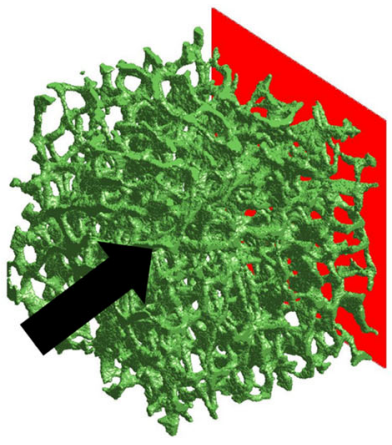

(b)

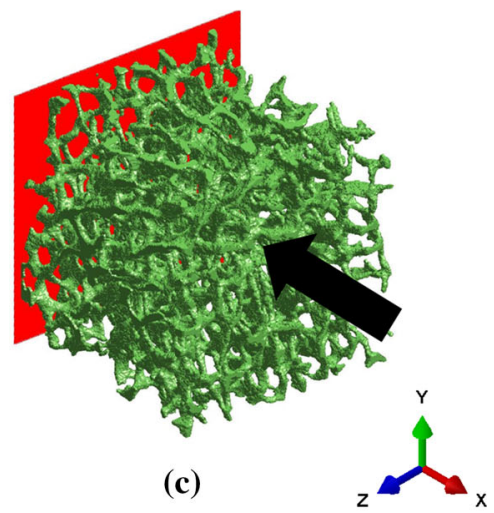

(c)

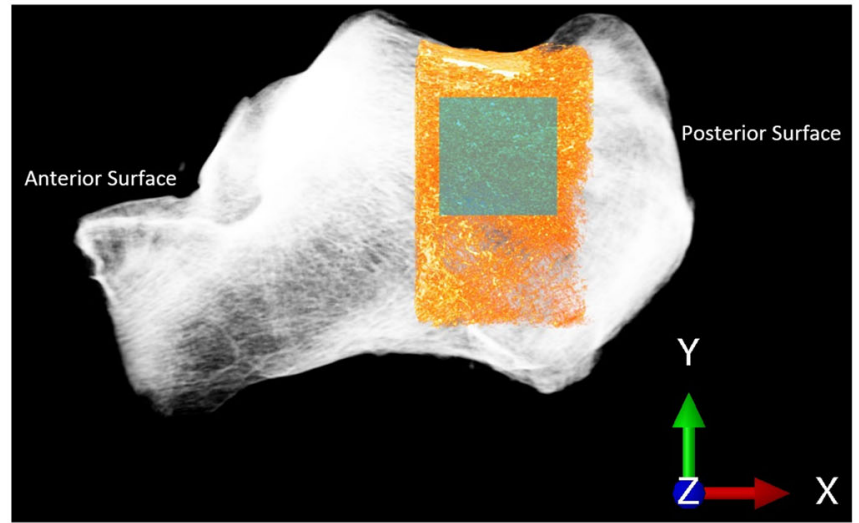

(d)

Fig. 3 Illustrative representation of three compressive loading scenarios: a loading case 1 , b loading case 2 and $\mathbf{c}$ loading case 3 . The loading-side elemental nodes (indicated by the black arrows) were assigned to compressive uniaxial displacement and suppressed in the other directions, where the elemental nodes on the opposite side were fully suppressed in all directions (indicated by the red surfaces). $\mathbf{d}$ The position of the selected microstructures (light blue) with the calcaneus bone

The BV/TV values for the five cadaveric specimens are relatively scattered $(8.70 \pm 4.90 \%, 3.80-14.31 \%)$ where similar observation is also reported by Ulrich et al. (1995) for the calcaneus site. Specimens 1 and 5 have relatively lower $\mathrm{BV} / \mathrm{TV}$ values compared to the other specimens (Fig. 5). The effect of integration time was almost negligible between 100 and $200 \mathrm{~ms}$, except for HR-pQCT $\mathrm{TH}_{\mathrm{TH}}$. BV/TV is similar for microCT, microCTRes and HR-PQCT $\mathrm{TH}_{\mathrm{T} 1}$, whereas the $\mathrm{BV} / \mathrm{TV}$ of $\mathrm{HR}-\mathrm{pQCT} \mathrm{TH}_{\mathrm{T} 2}$ is $2-3$ times higher than the other values.

On average, the specimens $1-4$ had DA $=0.66 \pm 0.03$, whereas for specimen 5 (highly osteoporotic) DA was equal to 0.99. Also, the averaged angular difference between the main trabecular direction and the $x$-axis (see Fig. 3) was $13.57 \pm 3.80^{\circ}$ for specimens $1-4$ and $72.24^{\circ}$ for specimen 5 .

By investigating the plateness/rodness $\left(C_{\mathrm{r}}\right)$ distribution curves of MicroCT models, it can be noted that the local geometry of rod/plate-like members is well estimated since the area under the curves for $C_{\mathrm{r}}<0$ is significantly larger for the less-dense specimens 1 and 5 (see specimen 1 in Fig. 6 and specimens 2-5 in "Appendix 1") due to higher propor- 


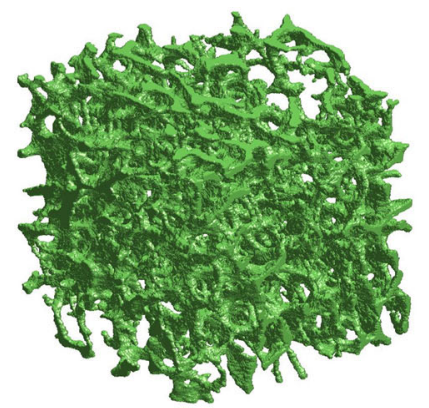

(a)

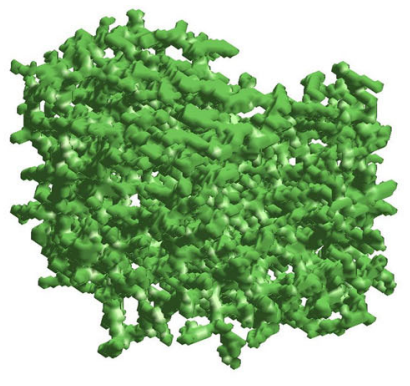

(b)

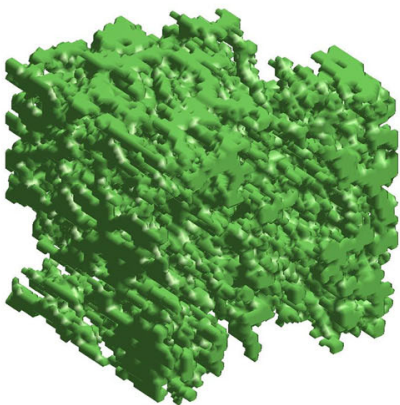

(c)

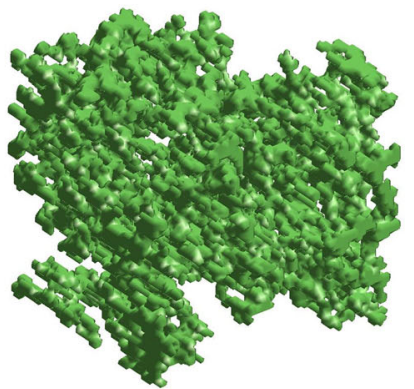

(d)

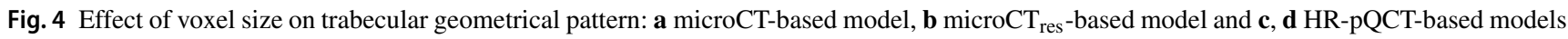
(integration time $=100 \mathrm{~ms}$ ) with minimum thresholds TH2 and TH1, respectively

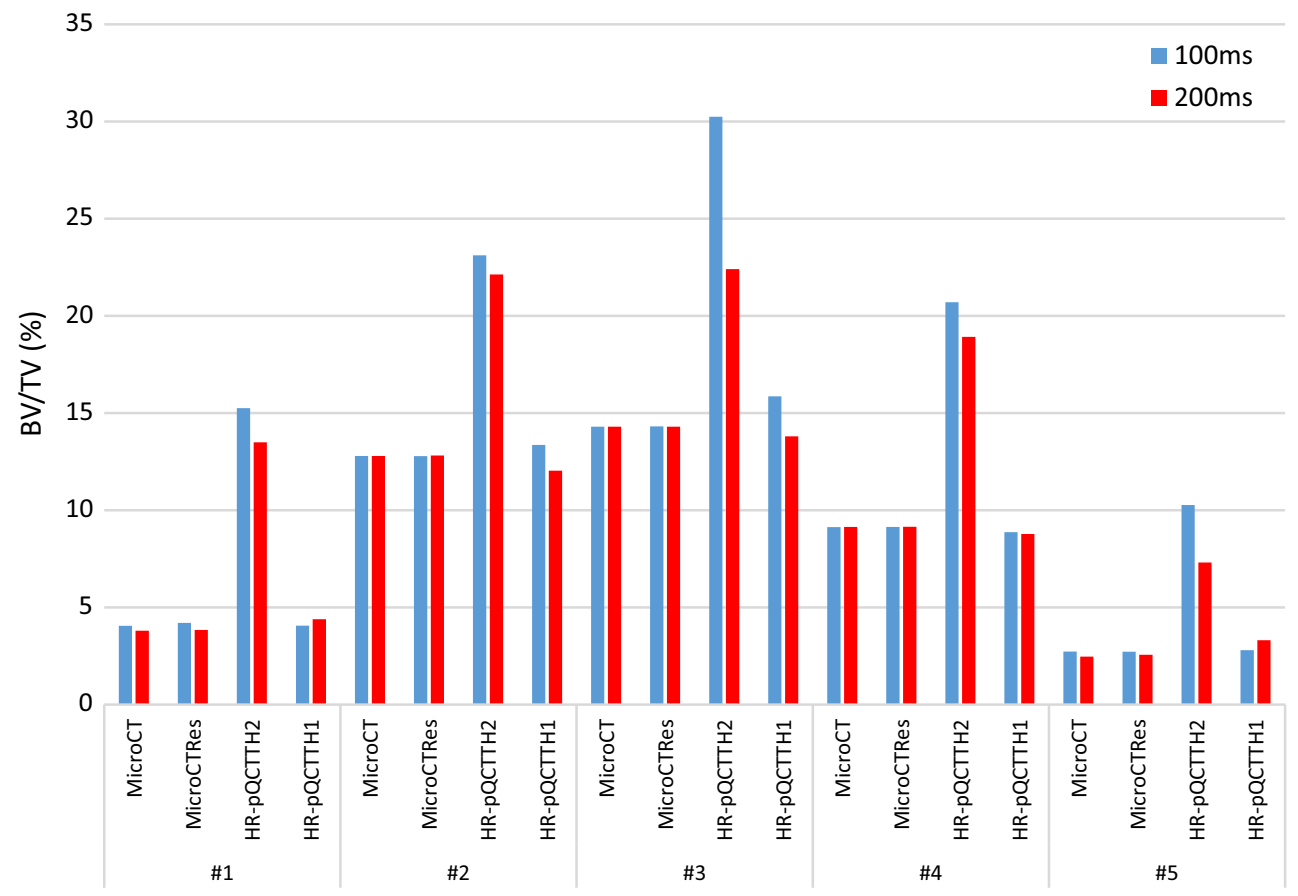

Fig. $5 \mathrm{BV} / \mathrm{TV}(\%)$ values for all specimens under microCT, microCT $\mathrm{T}_{\mathrm{Res}}, \mathrm{HP}_{\mathrm{pQCT}} \mathrm{TH}_{2}$ and $\mathrm{HR}-\mathrm{pQCT} \mathrm{TH}_{\mathrm{T} 1}$ for the integration time of 100 and $200 \mathrm{~ms}$

tion of rod-like trabeculae compared to specimens 2,3 and 4. However, unlike the microCT models, microCT $\mathrm{T}_{\mathrm{Res}}$ and HR$\mathrm{pQCT}_{\mathrm{TH} 1}$ microstructures show significantly overestimated distributions for rod-like members for all the cases. Considering HR-pQCT $\mathrm{TH}_{\mathrm{TH}}$, the reduction of threshold value has an adverse effect on the local morphologies for specimens with lower BV/TV (i.e. 1 and 5) where more members are identified as plate-like components through $C_{\mathrm{r}}$ distribution curves. Also, for all five specimens including the data from scans performed with the two integration times, the $C_{\mathrm{r}}$ distributions of HR-pQCT $\mathrm{TH}_{\mathrm{TH}}$ models, in general, are more similar to their equivalent microCT $\mathrm{T}_{\text {Res }}$.
For all five specimens, the microCT $\mathrm{T}_{\mathrm{Res}}$ and HR$\mathrm{pQCT}_{\mathrm{TH} 1 / \mathrm{TH} 2}$ models have significantly larger $S_{\text {thick }}$ compared to their corresponding gold-standard microCT models considering both integration times (Fig. 7).

\subsection{Mechanical analysis}

The average von Mises stress (mean of microFE elements von Mises stress values) was similar between microCT and HRpQCT $_{\mathrm{TH} 2}$ models (loading condition 1, Fig. 8 and loading conditions 2 and 3, "Appendix 2"). MicroCT Res $_{\text {and HR- }}$ $\mathrm{pQCT}_{\mathrm{TH} 1}$ models have $2-3$ times lower stress, and both are similar. 
(a) $100 \mathrm{~ms}$

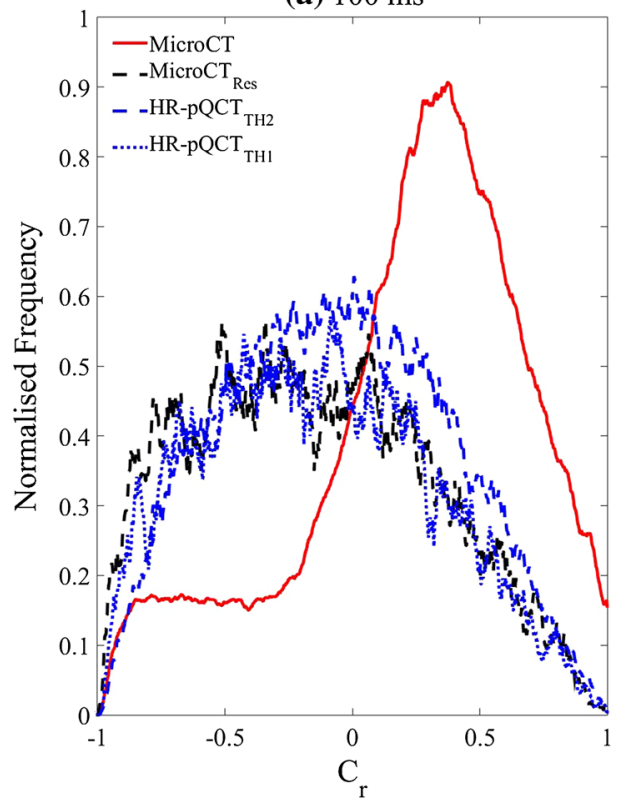

(b) $200 \mathrm{~ms}$

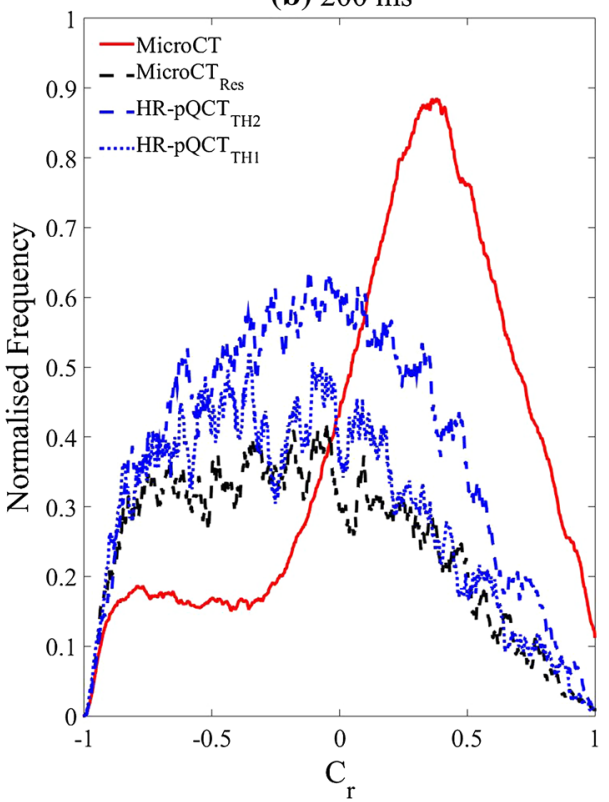

Fig. 6 Sample 1, plateness/rodness distribution for HR-pQCT models with integration times a $100 \mathrm{~ms}$ and $\mathbf{b} 200 \mathrm{~ms}$ and their counterparts registered microCT and microC $\mathrm{T}_{\text {Res }}$ models

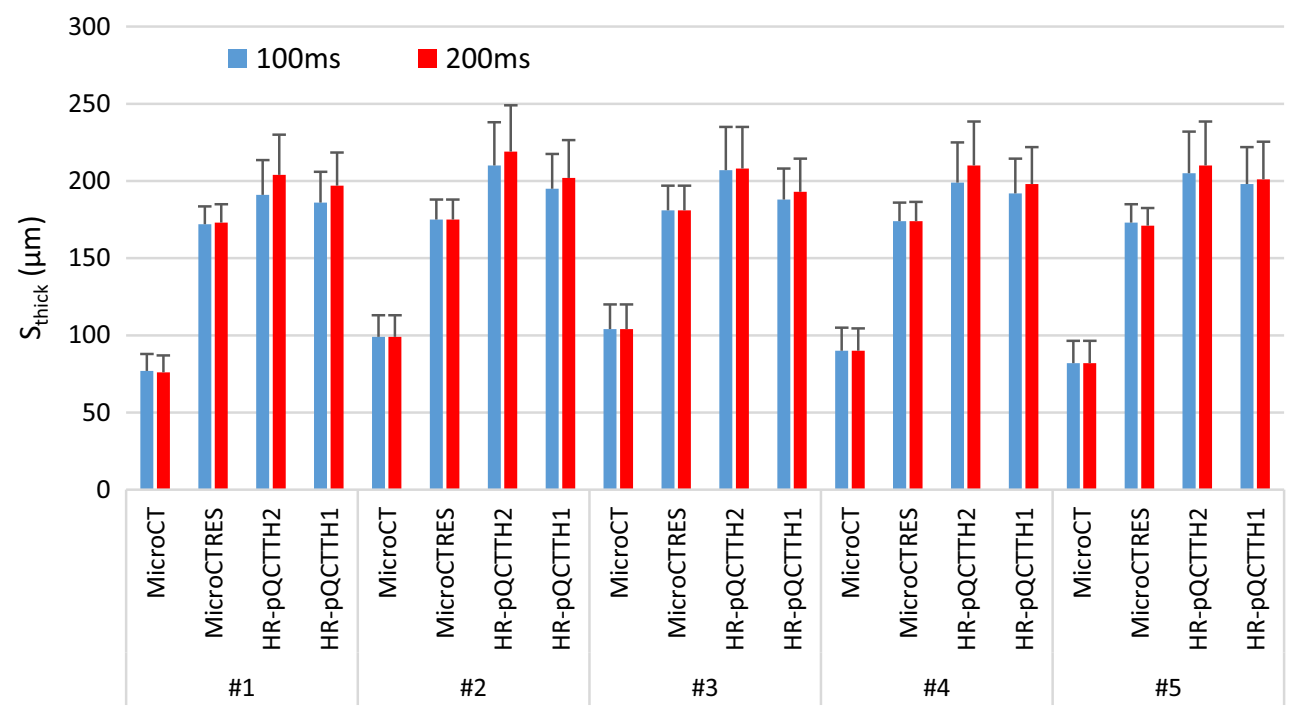

Fig. 7 Averaged structural thickness values for microCT, microCT $\mathrm{Res}_{\mathrm{R}}, \mathrm{HR}-\mathrm{pQCT} \mathrm{T}_{\mathrm{TH} 1}$ and $\mathrm{HR}-\mathrm{pQCT} \mathrm{T}_{\mathrm{TH} 2}$ models for scanning integration times of 100 and $200 \mathrm{~ms}$

The total reaction force (equivalent to apparent stiffness) was overestimated by the HR-pQCT $\mathrm{T}_{\mathrm{TH} 2}$ model (except for sample 4), whereas the microCT $\mathrm{Res}_{\mathrm{Res}}$ and $\mathrm{HR}-\mathrm{pQCT}_{\mathrm{TH} 1}$ models significantly underestimated it (loading condition 1, Fig. 9 and loading conditions 2 and 3, "Appendix 2"). A large variability was also found from one specimen to another.

By considering these five sample cases, the HR-pQCT $\mathrm{T}_{\mathrm{TH} 2}$ model results in significantly overestimated $F_{\text {frct }}$ values, especially for low-BV/TV samples (\#1 and \#5); see Fig. 10 for the loading condition 1 and "Appendix 2" for the loading condition 2 and 3 . The HR-pQCT $\mathrm{TH}_{\mathrm{TH}}$ model underestimated the failure load and was similar to microCT $\mathrm{T}_{\text {Res }}$ model.

\section{Discussion}

Ex vivo mechanical finite element and microstructural analyses were conducted on HR-pQCT scans of calcaneum 


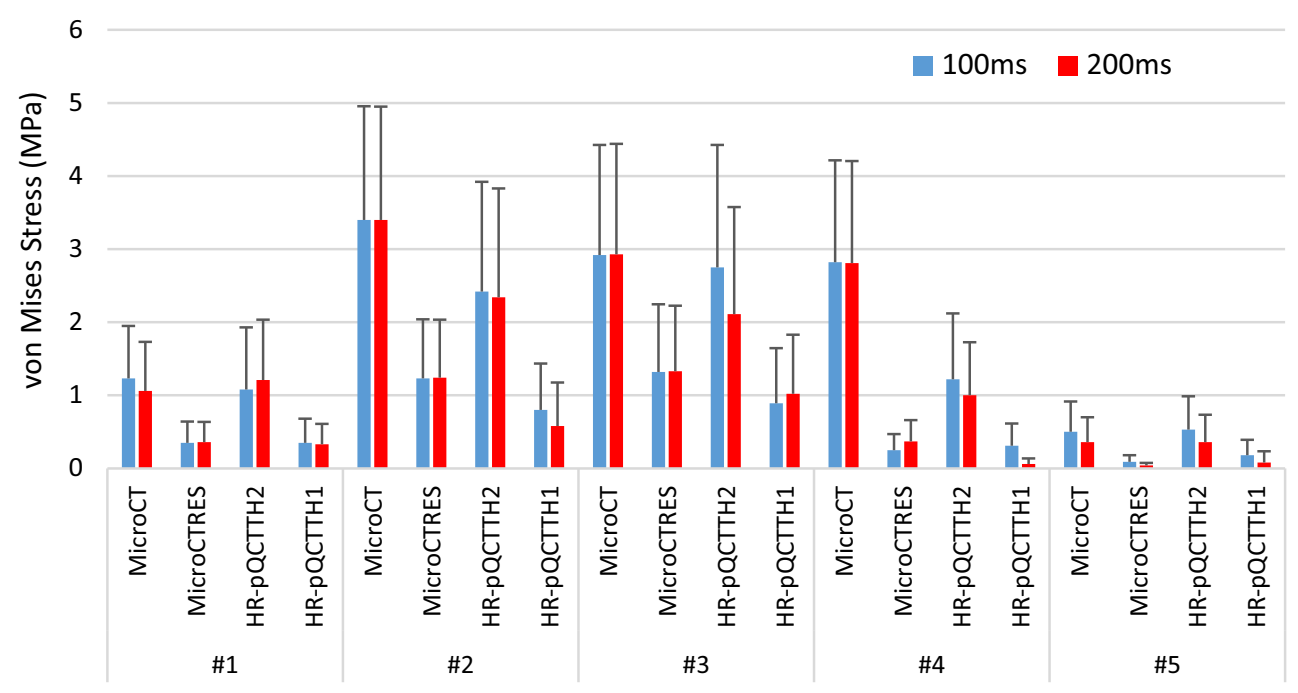

Fig. 8 Averaged and standard deviation von Mises stress for microCT, microCT $\mathrm{Res}_{\mathrm{R}}, \mathrm{HR}_{-\mathrm{pQCT}} \mathrm{TH1}$ and $\mathrm{HR}$-pQCT $\mathrm{TH}_{2}$ models for scanning integration times of 100 and $200 \mathrm{~ms}$ and for loading condition 1

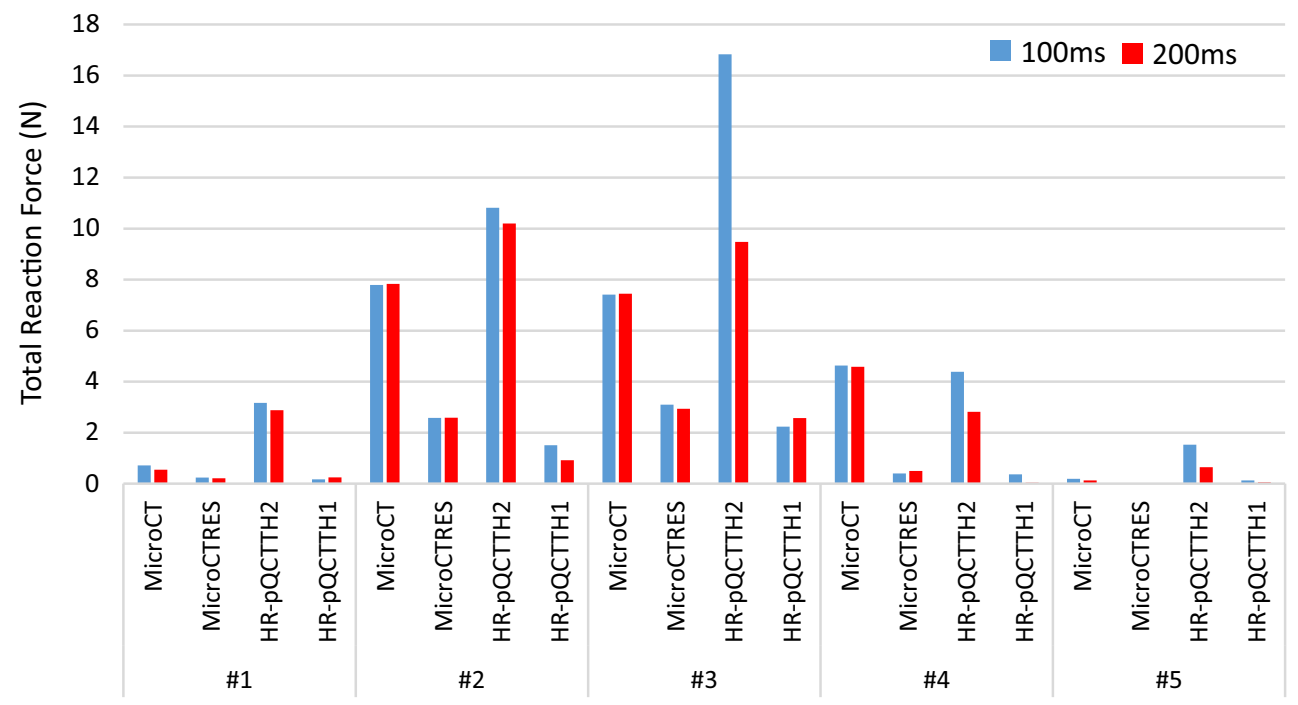

Fig. 9 Total reaction force for microCT, microCT $\mathrm{Res}_{\mathrm{Re}}$, HR-pQCT $\mathrm{TH}_{\mathrm{TH}}$ and $\mathrm{HR}-\mathrm{pQCT} \mathrm{T}_{\mathrm{TH} 2}$ models for scanning integration times of 100 and $200 \mathrm{~ms}$ and for the loading condition 1

trabecular subvolumes with microCT-based models used as gold standard. For these five sample cases, the local structural thickness, $S_{\text {thick }}$, of generated microCT $\mathrm{T}_{\text {Res }}$ and HR$\mathrm{pQCT}_{\mathrm{TH} 1 / \mathrm{TH} 2}$ models is significantly different from the goldstandard microCT model, whereas this difference is lower for microCT $\mathrm{T}_{\text {Res }}$ models. The inaccurate estimation of trabecular thickness by using XtremeCT I system is consistent with the results from Manske et al. (2015) for human cadaveric radii samples. It was found that increasing the integration time for HR-pQCT scans does not noticeably improve the geometrical quality of generated microstructures; as a result, their mechanical properties were also less improved. Also, $C_{\mathrm{r}}$ curves similarities between HR-pQCT $\mathrm{TH}_{\mathrm{TH}}$ and microCT $\mathrm{T}_{\mathrm{Res}}$ models highlight the dominant role of voxel sizes on the accuracy of morphological properties, since the microCT $\mathrm{T}_{\mathrm{Res}}$ is generated purely through downsampling method. A similar trend was observed between HR-pQCT $\mathrm{T}_{\mathrm{TH} 1}$ and microCT $\mathrm{T}_{\mathrm{Res}}$ FE results, which again shows a principal influence of voxel size on the quality of image-based microFE models.

The calculated stiffness of the selected regions of interest in calcaneus parts has an anisotropic property with highest and lowest stiffness in loading directions 3 and 2, respectively (see Fig. 3). It was also found that the averaged angular difference between the main trabecular direction and the $x$-axis is $13.57 \pm 3.80^{\circ}$ which is fairly alongside the third loading direction, resulting in maximum stiffness compared to the 


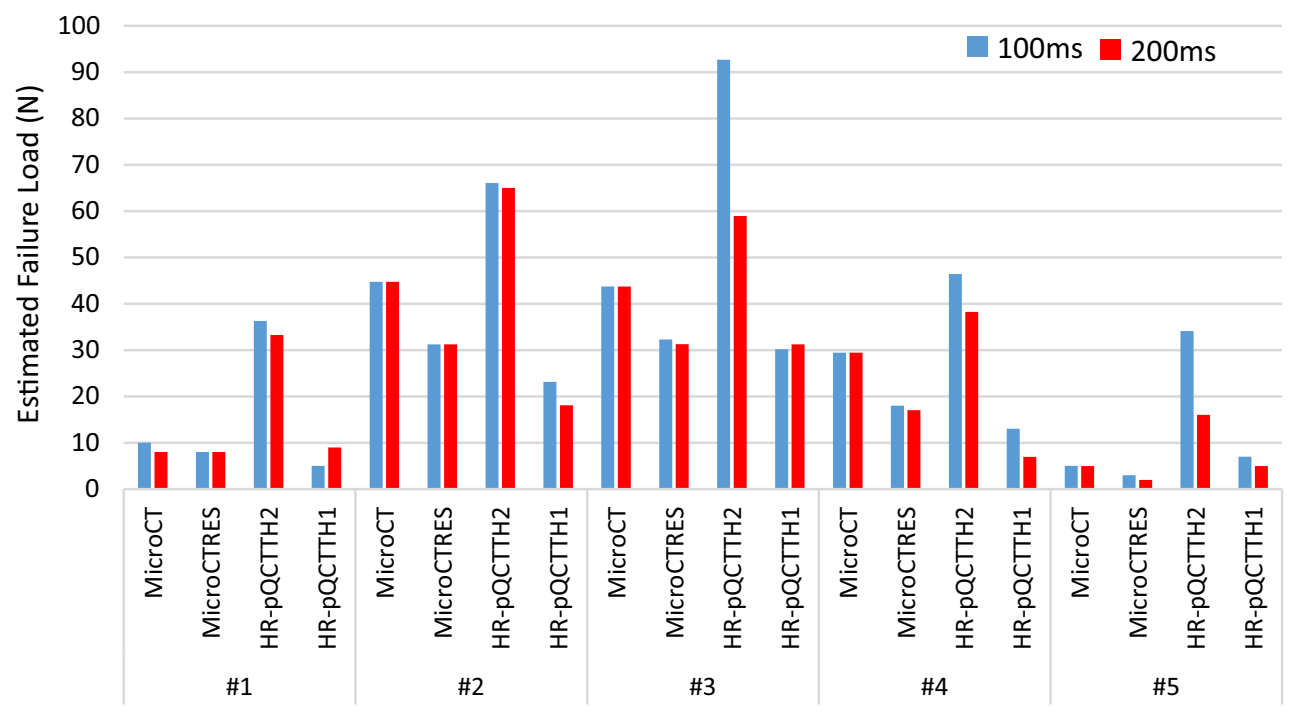

Fig. 10 Averaged estimated failure load for microCT, microCT $\mathrm{T}_{\mathrm{Res}}, \mathrm{HR}-\mathrm{pQCT}_{\mathrm{TH} 1}$ and $\mathrm{HR}-\mathrm{pQCT} \mathrm{T}_{\mathrm{TH} 2}$ models for scanning integration times of 100 and $200 \mathrm{~ms}$ and for the loading condition 1

other loading cases. It is noteworthy that this anisotropic mechanical response is also observed in HR-pQCT and resampled-based microstructures as well. Therefore, it can be stated that the HR-pQCT $\mathrm{TH}_{\mathrm{TH}}$ models are potentially able to catch anisotropic mechanical response, according to the trabecular orientations.

One of the main objectives of this work was to perform comparative study on failure load prediction, $F_{\text {frct }}$, in the light of the imaging/modelling technique. From both geometrical analysis and FE results, it was found that the single value threshold technique does not provide accurate image-based trabecular microFE model with voxel size of $82 \mu \mathrm{m}$. Using HR-pQCT-based microstructure with overestimated BV/TV value (i.e. $\mathrm{HR}-\mathrm{pQCT} \mathrm{T}_{\mathrm{TH} 2}$ ), the $\mathrm{Stress}_{\mathrm{vm}}$ was similar to the microCT model. However, it overestimated the apparent stiffness (measured as $F_{\text {tot }}$ ). It should be pointed out however, it was assumed that the bone elastic modulus is identical for all the samples and independent from the structural and physiological properties.

The microCT models have different $S_{\text {thick }}$ to HR-pQCT. This means, for microFE models which are generated from $82 \mu \mathrm{m}$ voxel size images, there is a wide image greyscale distribution. In contrast, for higher-resolution microCT images, the smaller voxel size will result in narrower greyscale distribution. Therefore, using single value threshold technique is more efficient for images with narrow intensity distribution such as cases with small voxel size or homogenous geometry (e.g. cortical bone). It is important to note that the (Pistoia et al. 2002) criteria have been established based on axial loading on the distal radius where cortical bone encloses the trabecular region. From several FE analyses on distal radius fracture (e.g. HR-pQCT with $82 \mu \mathrm{m}$ resolu- tion, Vilayphiou et al. 2010), it is reported that the cortical part of the distal radius bears more load (i.e. stress) than the trabecular region and therefore acts as mechanical stabiliser by transmitting force into applied loading direction. Also, due to almost homogenous geometry of cortical bone, this region has narrow image greyscale distribution where using single value threshold technique results in accurate cortical microFE. Therefore, potential inaccurate mechanical predictions resulting from the impact of large voxel size on trabecular microFE were masked by the cortical dominance. In our study, the use of purely trabecular calcaneus microFE models confirms that HR-pQCT $\mathrm{TH}_{\mathrm{TH}}$ models do not sufficiently represent the accurate microstructure geometry, especially for specimens with low BV/TV (e.g. sample 5). In particular for such low-BV/TV samples, there were weak trabecular connectivities due to their insufficient voxel resolution; therefore, performing the single level threshold method resulted in the elimination of large amounts of microstructure that were visually realised (for example, see Fig. 4).

Although only five calcaneum cadaveric samples were used in this study, high variability in terms of architecture was found from one sample to another and yet the mechanical analysis was fairly consistent for all samples providing confidence that the main outcomes of this study are valid for any calcaneum trabecular bones.

\section{Conclusions}

Overall it was found that the morphology of XtremeCT I HR-pQCT $\mathrm{TH}_{\mathrm{TH}}$ models for calcaneus trabecular bone is sig- 
nificantly different to their corresponding microCT models. Due to wide greyscale distribution of HR-pQCT images, the final HR-pQCT-based microFE is very sensitive to single level threshold technique where the whole morphology and $\mathrm{BV} / \mathrm{TV}$ can be affected by the chosen threshold. By analysing microCT $_{\text {Res }}$ and HR-pQCT $\mathrm{TH}_{\mathrm{TH}}$ models and both morphological and mechanical similarities between the two, it was found that voxel size has a dominant effect on the microFE quality when the single value threshold method is used for generating voxel-based microstructure. The mechanical simulation results showed significant difference between the predictions of gold-standard microCT and XtremeCT I HR-pQCT ${ }_{\mathrm{TH} 1}$ models that in average are $70 \%$ lower for von Mises stress, $64 \%$ lower for apparent stiffness and 33\% lower for failure load prediction.

In conclusion, a single threshold value cannot predict both morphological structures (BV/TV) and biomechanical analyses (stress, apparent stiffness and failure load prediction) correctly. Since the resolution of XtremeCT I is not sufficient to resolve trabecular thickness, caution should be made to use it to monitor the effect of the progression of the disease or the effect of a bone drug if the target is, for example, bone remodelling since accurate trabecular thickness will not be achieved. Alternatively, employing more advanced segmentation strategies (e.g. local adaptive threshold method Burghardt et al. 2007) may improve the accuracy of HRpQCT-based microstructures; nevertheless, a comprehensive geometrical and mechanical evaluation study is still required,

(a) $100 \mathrm{~ms}$

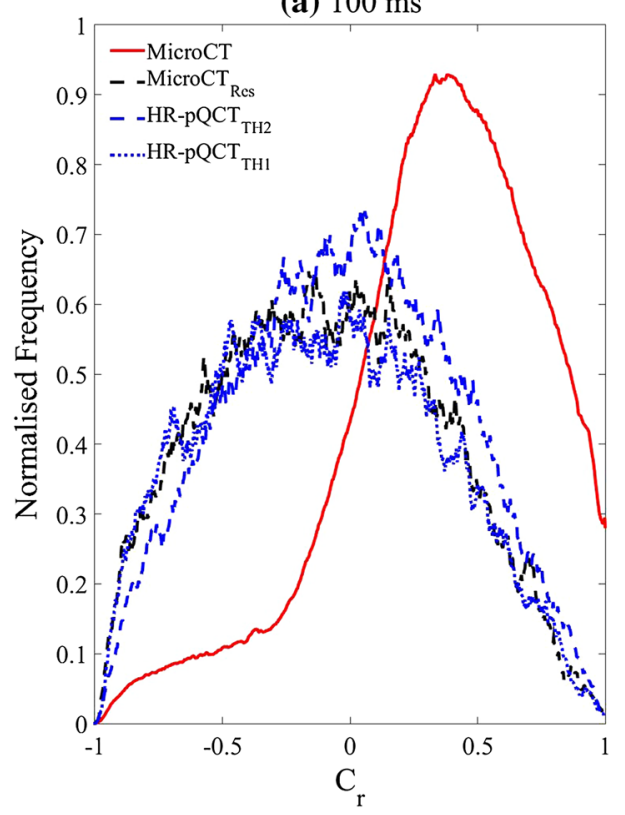

in particular for the trabecular regions with small BV/TV values.

Acknowledgements The authors acknowledge Margaret Paggiosi for operating the HR-pQCT scanner and the Skelet.Al Lab (in particular Kirsty Nicholson) for the help with the MicroCT scans. Funding from EPSRC (EP/K03877X/1), MRC and Arthritis Research UK as part of the MRC-Arthritis Research UK Centre for Integrated Research into Musculoskeletal Ageing (CIMA) and HEFCE Catalyst fund are acknowledged.

Funding This study was funded by EPSRC (EP/K03877X/1).

\section{Compliance with ethical standards}

Conflict of interest The authors declare that they have no conflict of interest.

Open Access This article is distributed under the terms of the Creative Commons Attribution 4.0 International License (http://creativecomm ons.org/licenses/by/4.0/), which permits unrestricted use, distribution, and reproduction in any medium, provided you give appropriate credit to the original author(s) and the source, provide a link to the Creative Commons license, and indicate if changes were made.

\section{Appendix 1}

See Figs. 11, 12, 13 and 14.

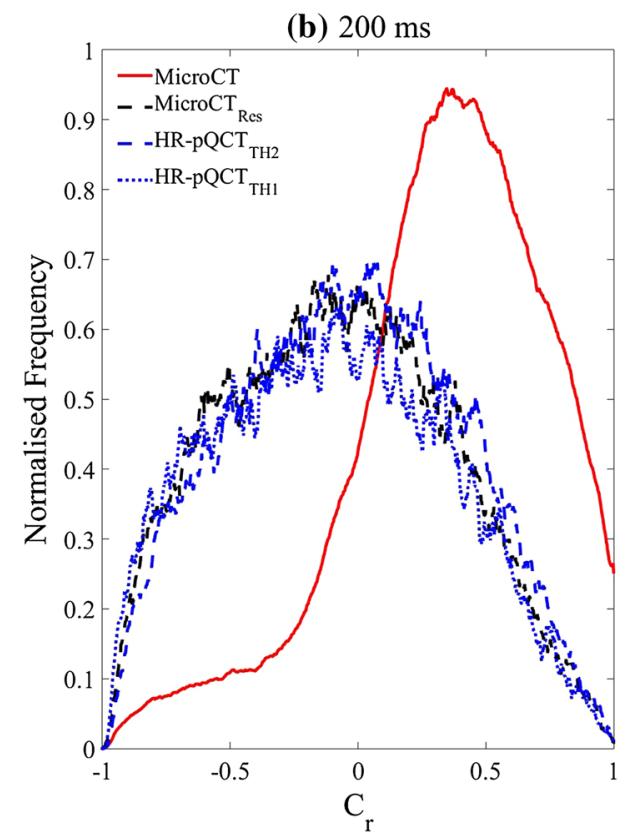

Fig. 11 Sample 2, plateness/rodness distribution for HR-pQCT models with integration times a $100 \mathrm{~ms}$ and b $200 \mathrm{~ms}$ and their counterparts registered microCT and microCT $T_{\text {Res }}$ models 
(a) $100 \mathrm{~ms}$

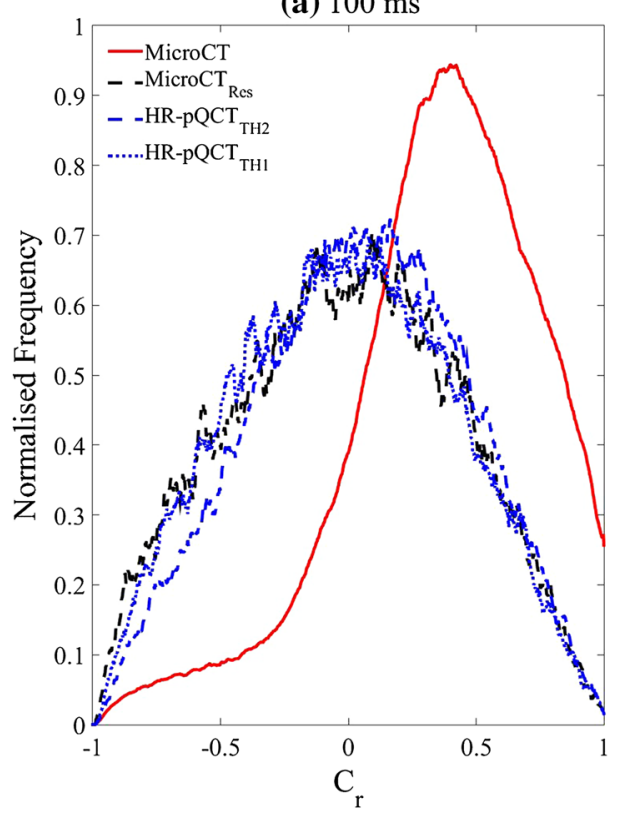

(b) $200 \mathrm{~ms}$

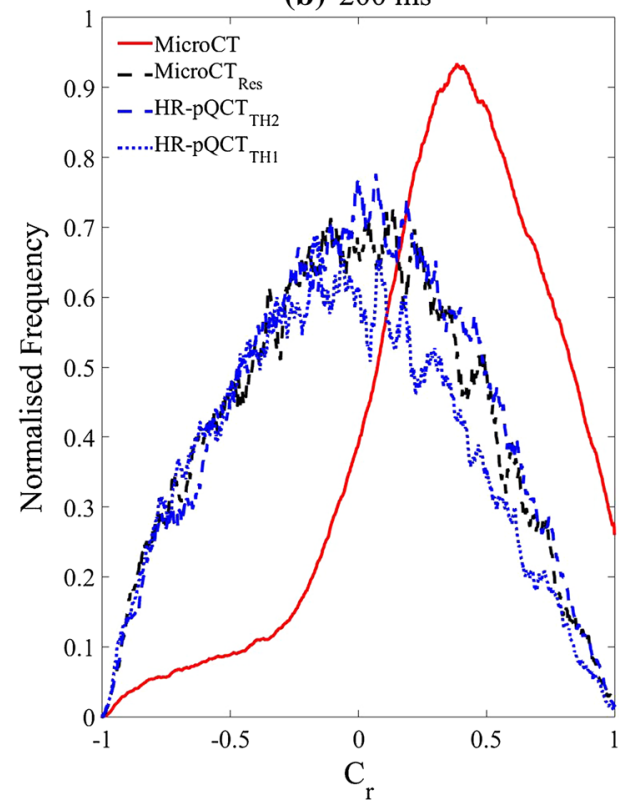

Fig. 12 Sample 3, plateness/rodness distribution for HR-pQCT models with integration times a $100 \mathrm{~ms}$ and b $200 \mathrm{~ms}$ and their counterparts registered microCT and microCT $T_{\text {Res }}$ models

(a) $100 \mathrm{~ms}$

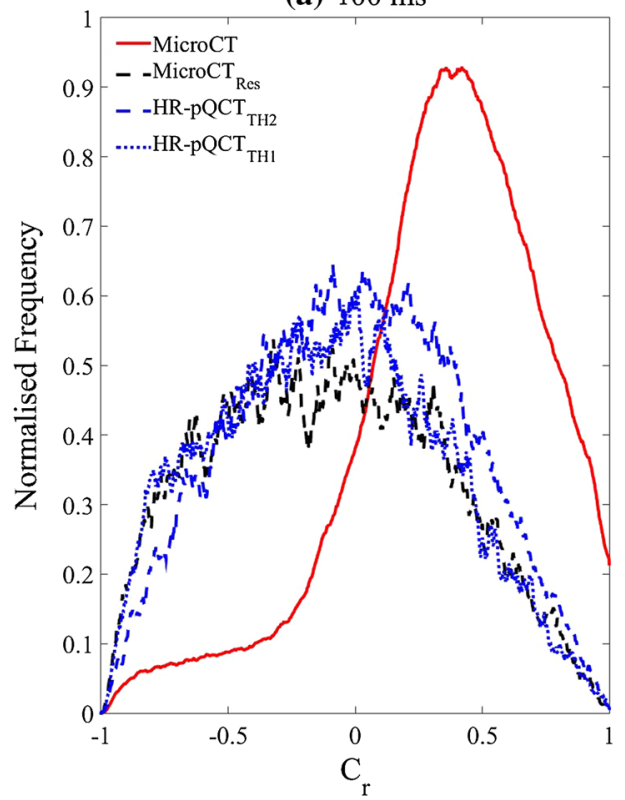

(b) $200 \mathrm{~ms}$

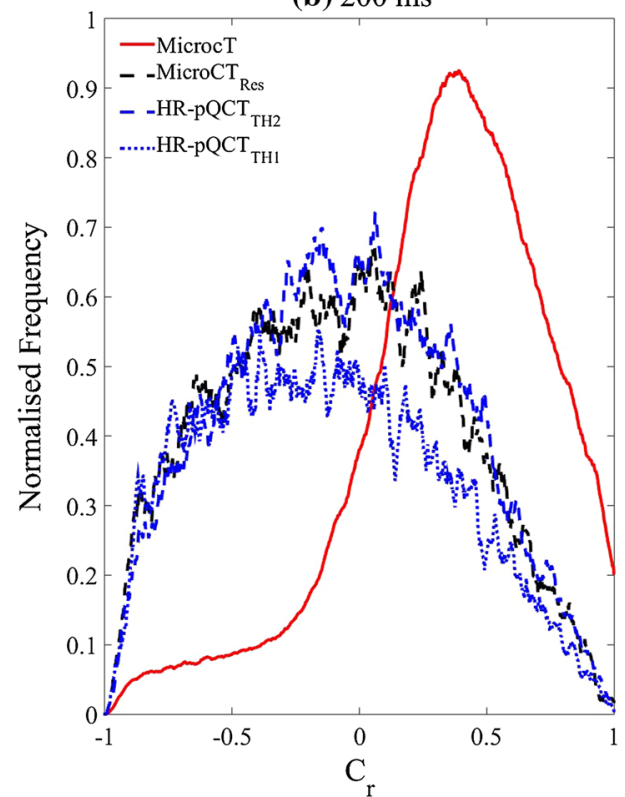

Fig. 13 Sample 4, plateness/rodness distribution for HR-pQCT models with integration times a $100 \mathrm{~ms}$ and b $200 \mathrm{~ms}$ and their counterparts registered microCT and microCT $T_{\text {Res }}$ models 
(a) $100 \mathrm{~ms}$

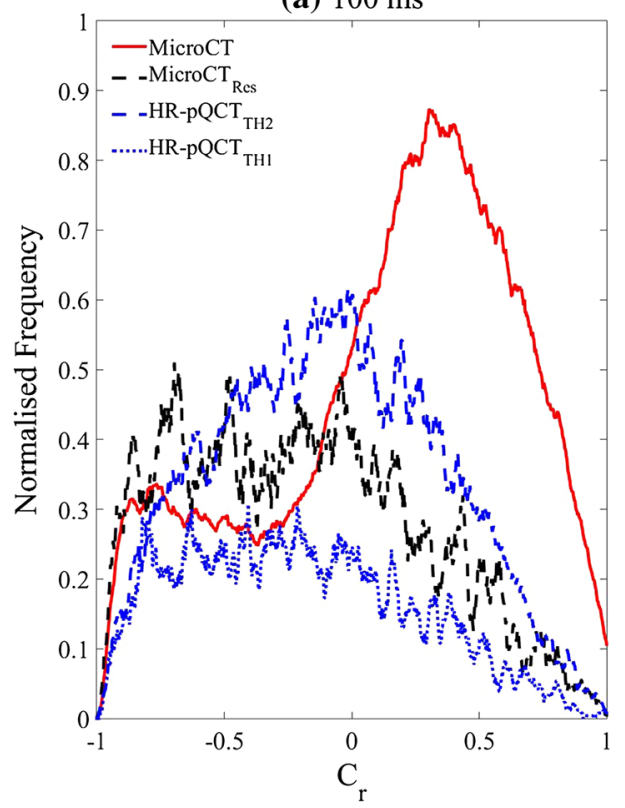

(b) $200 \mathrm{~ms}$

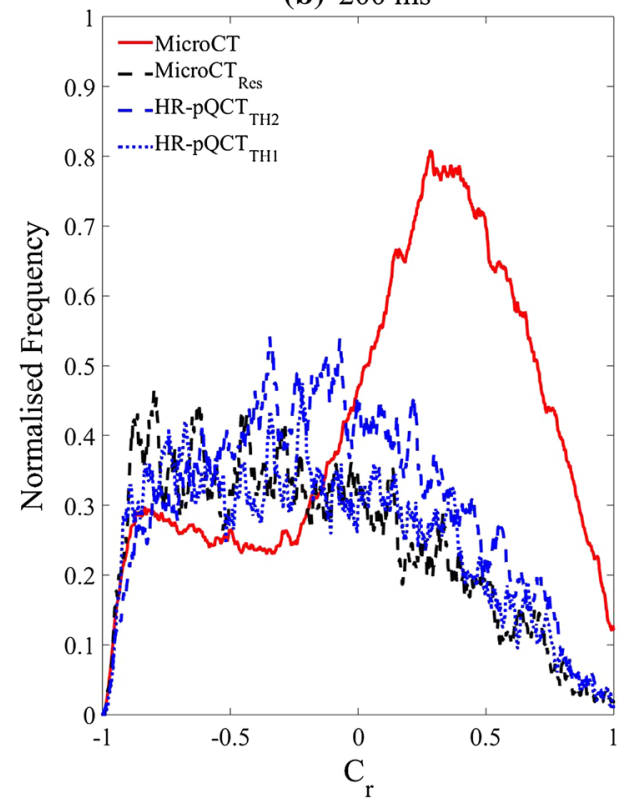

Fig. 14 Sample 5, plateness/rodness distribution for HR-pQCT models with integration times a $100 \mathrm{~ms}$ and b $200 \mathrm{~ms}$ and their counterparts registered microCT and microCT $T_{\text {Res }}$ models

\section{Appendix 2}

See Figs. 15, 16, 17, 18, 19 and 20.

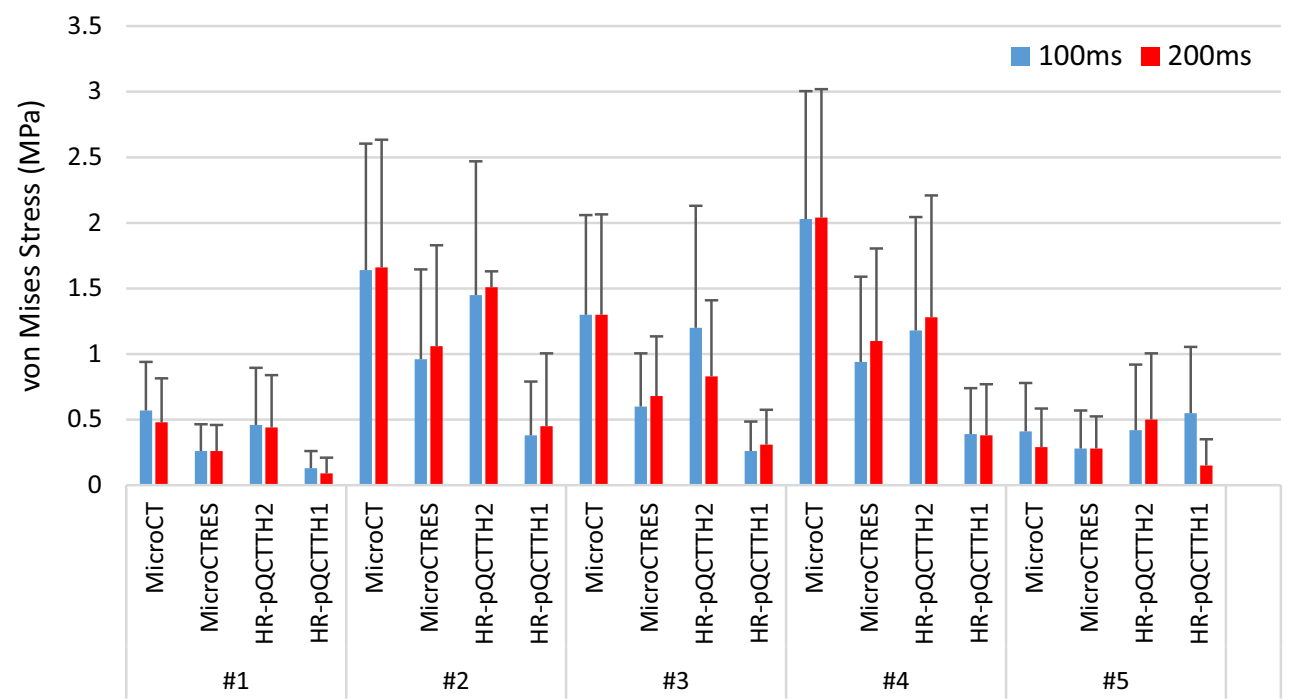

Fig. 15 Averaged and standard deviation von Mises stress for microCT, microCT $\mathrm{Res}_{\mathrm{R}}, \mathrm{HR}-\mathrm{pQCT} \mathrm{TH}_{\mathrm{TH}}$ and $\mathrm{HR}-\mathrm{pQCT}_{\mathrm{TH} 2}$ models for scanning integration times at 100 and $200 \mathrm{~ms}$ and for loading condition 2 


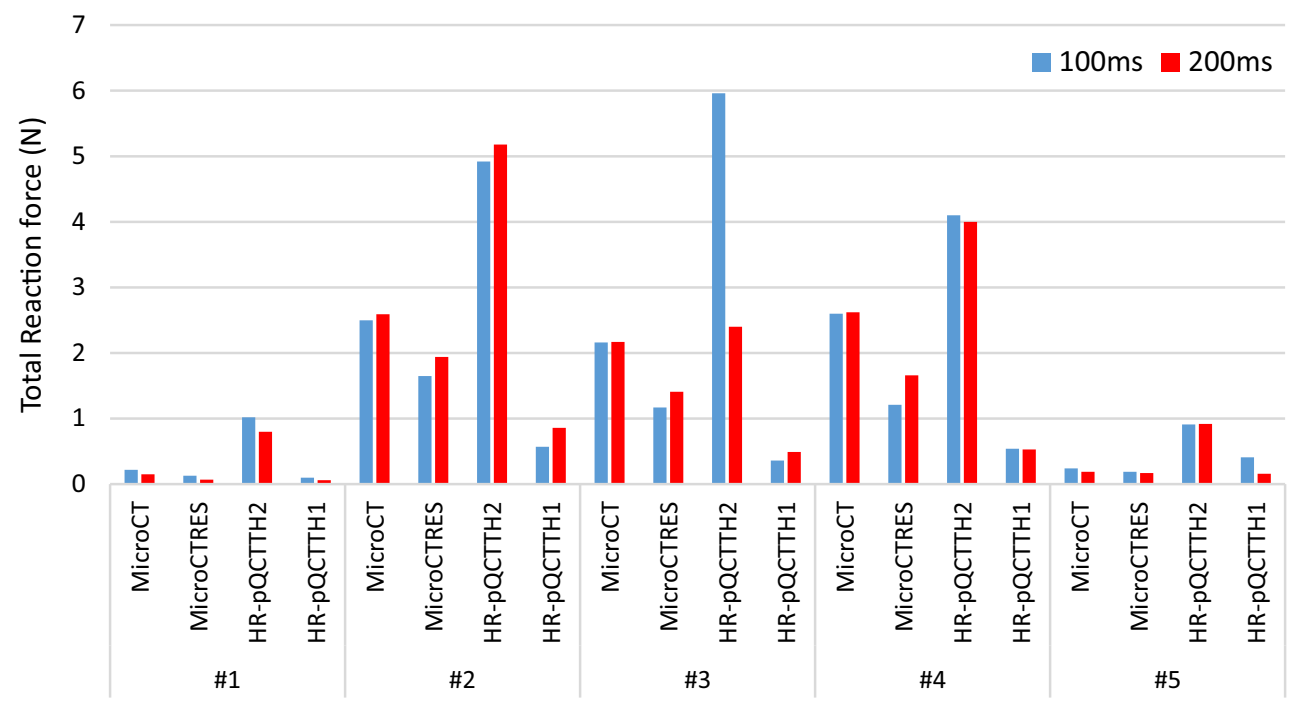

Fig. 16 Total reaction force for microCT, and microCT $\mathrm{Res}_{\mathrm{R}}, \mathrm{HR}_{-\mathrm{pQCT}} \mathrm{TH1}$ and $\mathrm{HR}-\mathrm{pQCT}_{\mathrm{TH} 2}$ models for scanning integration times at 100 and $200 \mathrm{~ms}$ and for the loading condition 2

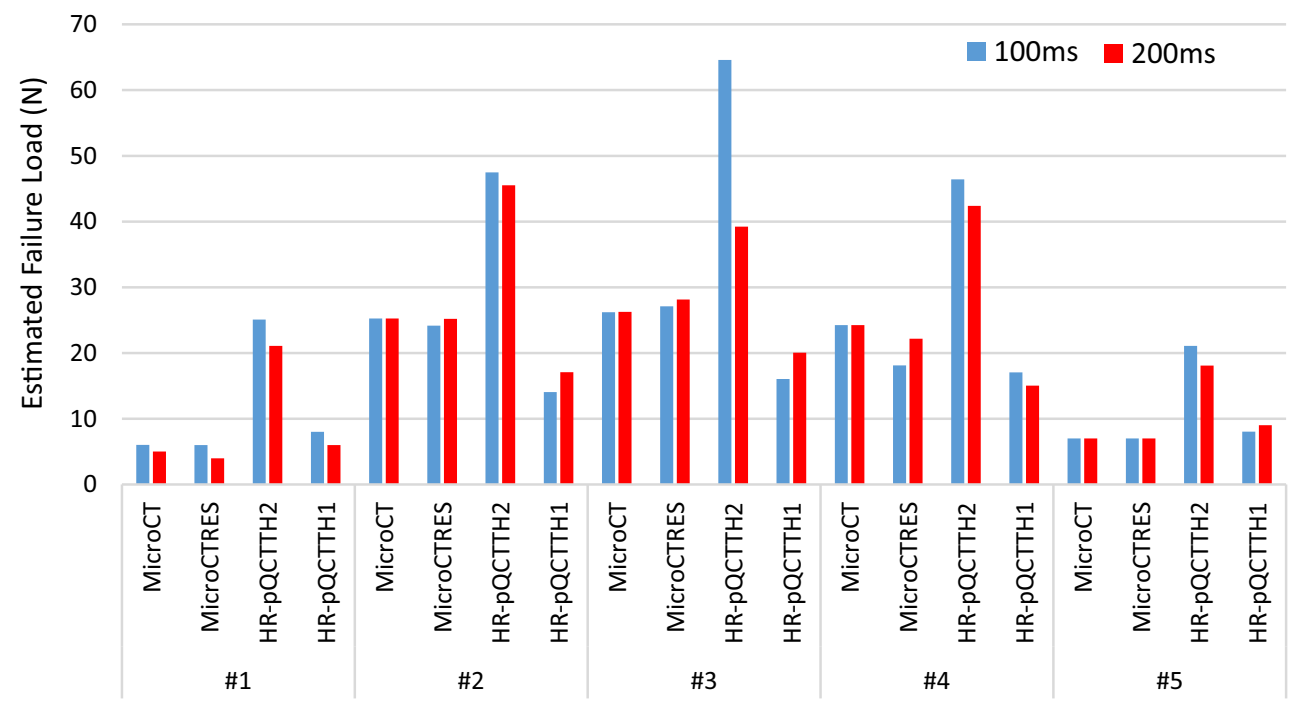

Fig. 17 Averaged estimated failure load for microCT, microCT $\mathrm{T}_{\mathrm{Res}}, \mathrm{HR}_{\mathrm{pQCT}} \mathrm{TH1}$ and $\mathrm{HR}-\mathrm{pQCT} \mathrm{T}_{\mathrm{TH} 2}$ models for scanning integration times of 100 and $200 \mathrm{~ms}$ and for the loading condition 2 


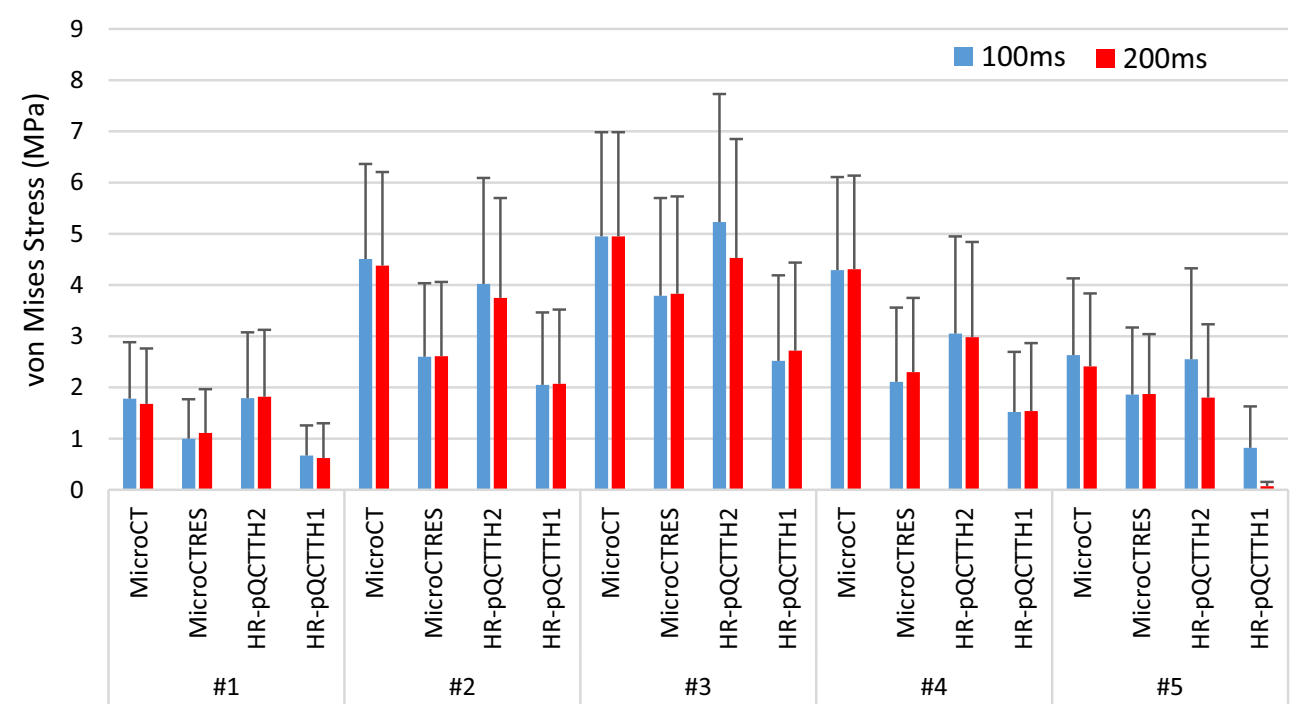

Fig. 18 Averaged and standard deviation von Mises stress for microCT, microCT $\mathrm{T}_{\mathrm{Res}}, \mathrm{HR}-\mathrm{pQCT} \mathrm{TH}_{\mathrm{T} 1}$ and $\mathrm{HR}_{\mathrm{pQCT}} \mathrm{TH}_{2}$ models for scanning integration times of 100 and $200 \mathrm{~ms}$ and for loading condition 3

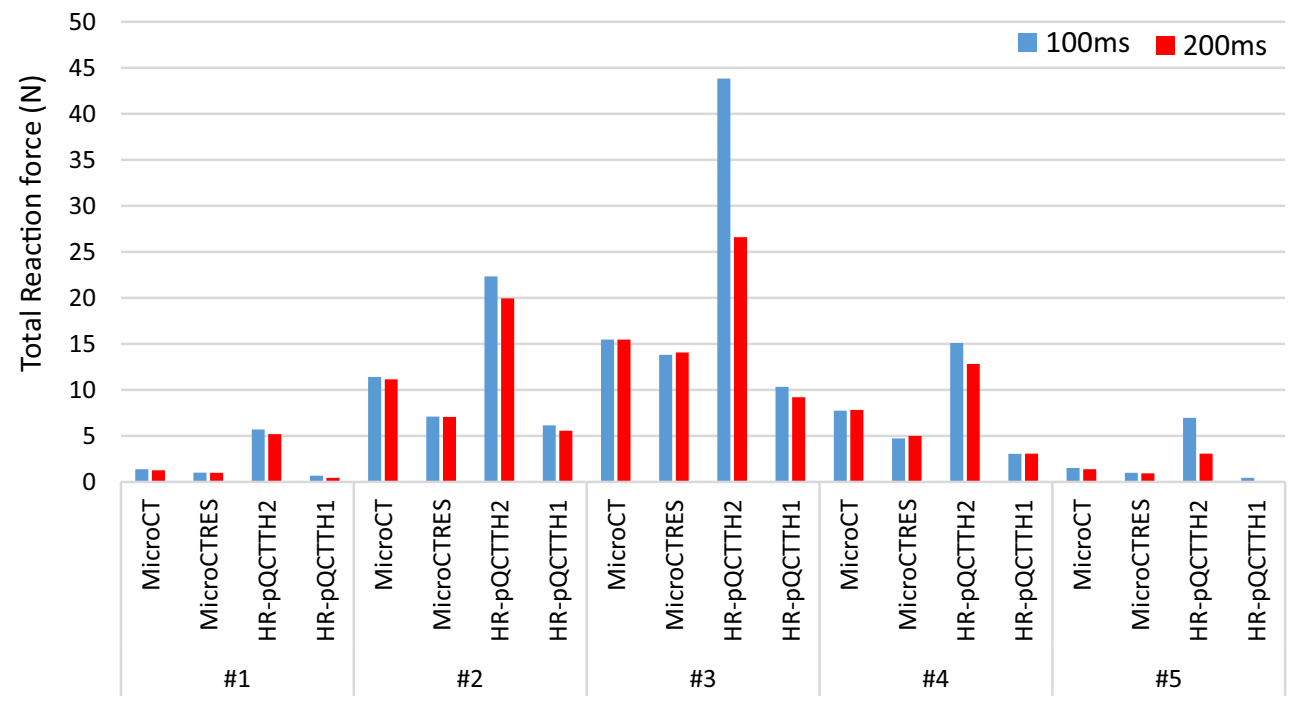

Fig. 19 Total reaction force for microCT, microCT $\mathrm{Res}_{\mathrm{Res}}, \mathrm{HR}_{-\mathrm{pQCT}} \mathrm{TH}_{\mathrm{T} 1}$ and $\mathrm{HR}-\mathrm{pQCT} \mathrm{TH}_{\mathrm{TH}}$ models for scanning integration times of 100 and $200 \mathrm{~ms}$ and for the loading condition 3 


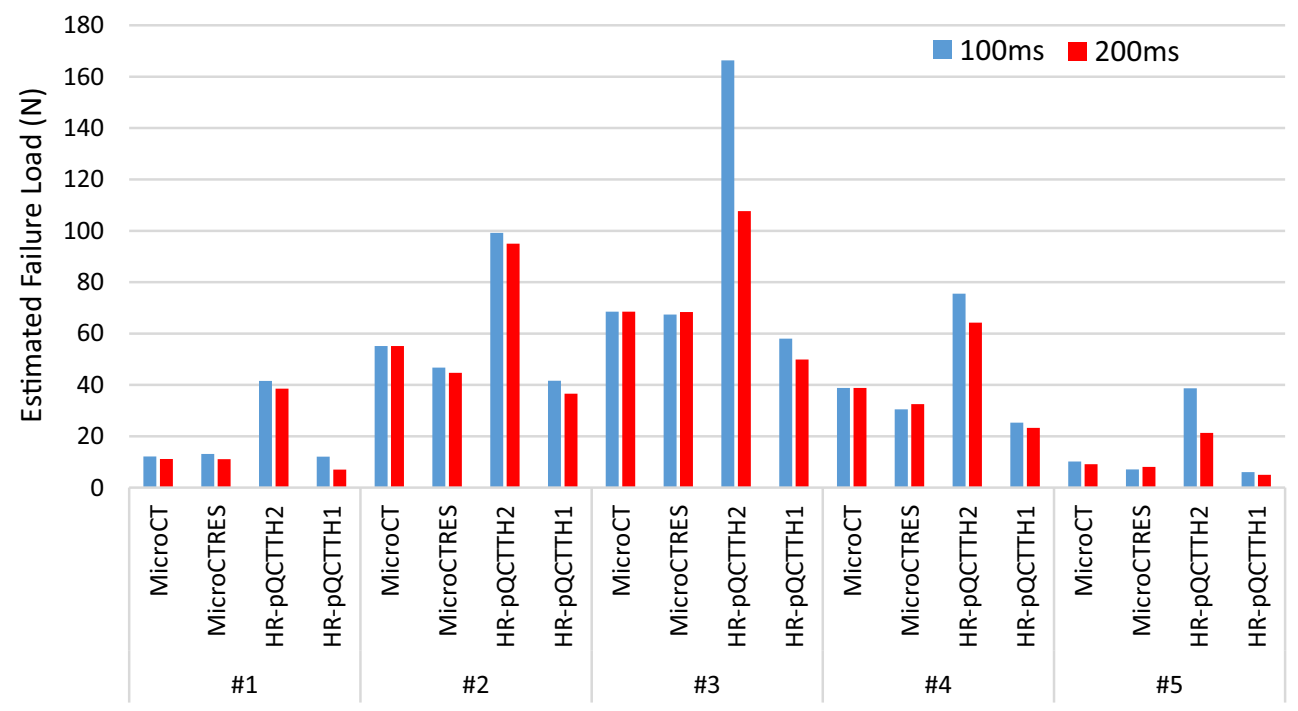

Fig. 20 Averaged estimated failure load for microCT, microCT $\mathrm{T}_{\mathrm{Res}}, \mathrm{HR}-\mathrm{pQCT}_{\mathrm{TH} 1}$ and $\mathrm{HR}-\mathrm{pQCT} \mathrm{T}_{\mathrm{TH} 2}$ models for scanning integration times of 100 and $200 \mathrm{~ms}$ and for the loading condition 3

\section{References}

Alberich-Bayarri A, Marti-Bonmati L, Perez MA, Sanz-Requena R, Lerma-Garrido JJ, Garcia-Marti G et al (2010) Assessment of 2D and 3D fractal dimension measurements of trabecular bone from high-spatial resolution magnetic resonance images at $3 \mathrm{~T}$. Med Phys 37(9):4930-4937

Bevill G, Keaveny TM (2009) Trabecular bone strength predictions using finite element analysis of micro-scale images at limited spatial resolution. Bone 44(4):579-584

Boutroy S, Van Rietbergen B, Sornay-Rendu E, Munoz F, Bouxsein ML, Delmas PD (2008) Finite element analysis based on in vivo HR-pQCT images of the distal radius is associated with wrist fracture in postmenopausal women. J Bone Miner Res 23(3):392-399

Burghardt AJ, Kazakia GJ, Majumdar S (2007) A local adaptive threshold strategy for high resolution peripheral quantitative computed tomography of trabecular bone. Ann Biomed Eng 35:1678-1686

Christen P, Schulte FA, Zwahlen A, van Rietbergen B, Boutroy S, Melton LJ et al (2016) Voxel size dependency, reproducibility and sensitivity of an in vivo bone loading estimation algorithm. J R Soc Interface 13(114):20150991

Depalle B, Chapurlat R, Walter-Le-Berre H, Bou-Said B, Follet H (2013) Finite element dependence of stress evaluation for human trabecular bone. J Mech Behav Biomed Mater 18:200-212

Ding M, Odgaard A, Hvid I (1999) Accuracy of cancellous bone volume fraction measured by micro-CT scanning. J Biomech 32:323-326

Enns-Bray WS, Ariza O, Gilchrist S, Soyka RPW, Vogt PJ, Palsson H et al (2016) Morphology based anisotropic finite element models of the proximal femur validated with experimental data. Med Eng Phys 38(11):1339-1347

Farr JN, Khosla S (2015) Skeletal changes through the lifespan-from growth to senescence. Nat Rev Endocrinol 11(9):513-521

Fuller H, Fuller R, Pereira RMR (2015) High resolution peripheral quantitative computed tomography for the assessment of morphological and mechanical bone parameters. Rev Bras Reumatol 55(4):352-362
k-Wave: a MATLAB toolbox for the time-doamin simulation of acoustic wave fields (2015). http://www.k-wave.org. Accessed 18 Nov 2015

Laib A, Ruegsegger P (1999) Comparison of structure extraction methods for in vivo trabecular bone measurements. Comput Med Imaging Graph 23(2):69-74

Liebl H, Baum T, Karampinos DC, Patsch J, Malecki A, Schaff F, Eggl E, Rummeny EJ, Pfeiffer F, Bauer JS (2014) Emerging research on bone health using high-resolution CT and MRI. Curr Radiol Rep 2:31

Liu XS, Sajda P, Saha PK, Wehrli FW, Bevill G, Keaveny TM, Guo XE (2008) Complete volumetric decomposition of individual trabecular plates and rods and its morphological correlations with anisotropic elastic moduli in human trabecular bone. J Bone Miner Res 23(2):223-235

Liu XS, Zhang XH, Sekhon KK, Adams MF, McMahon DJ, Bilezikian JP et al (2010) High-resolution peripheral quantitative computed tomography can assess microstructural and mechanical properties of human distal tibial bone. J Bone Miner Res 25(4):746-756

MacNeil JA, Boyd SK (2008) Bone strength at the distal radius can be estimated from high-resolution peripheral quantitative computed tomography and the finite element method. Bone 42(6):1203-1213

Manske SL, Zhu Y, Sandino C, Boyd SK (2015) Human trabecular bone microarchitecture can be assessed independently of density with second generation HR-pQCT. Bone 79:213-221

Manzanera A, Bernard T, Preteux F, Longuet B (1999) Medial faces from a concise 3D thinning algorithm. In: IEEE international conference on computer vision (ICCV'99), pp 337-343

McCloskey EV, Kanis JA, Oden A, Harvey NC, Bauer D, GonzalezMacias J, Hans D, Kaptoge S, Krieg MA, Kwok T, Marin F, Moayyeri A, Orwoll E, Gluer C, Johansson H (2015) Predictive ability of heel quantitative ultrasound for incident fractures: an individual-level meta-analysis. Osteoporos Int 26:1979-1987

Moisy F (2008) http://www.fast.u-psud.fr/ moisy/ml/. Accessed 18 Nov 2015

Nishiyama KK, Shane E (2013) Clinical imaging of bone microarchitecture with HR-pQCT. Curr Osteoporos Rep 11(2):147-155 
Paggiosi MA, Eastell R, Walsh JS (2014) Precision of high-resolution peripheral quantitative computed tomography measurement variables: influence of gender, examination site, and age. Calcif Tissue Int 94(2):191-201

Pialat JB, Vilayphiou N, Boutroy S, Gouttenoire PJ, Sornay-Rendu E, Chapurlat R, Peyrin F (2012) Local topological analysis at the distal radius by HR-pQCT: application to in vivo bone microarchitecture and fracture assessment in the OFELY study. Bone 51(3):362-368

Pistoia W, Van Rietbergen B, Lochmuller EM, Lill CA, Eckstein F, Ruegsegger P (2002) Estimation of distal radius failure load with micro-finite element analysis models based on three-dimensional peripheral quantitative computed tomography images. Bone 30(6):842-848

Putman MS, Milliren CE, Derrico N, Uluer A, Sicilian L, Lapey A, Sawicki G, Gordon CM, Bouxsein ML, Finkelstein JS (2014) Compromised bone microarchitecture and estimated bone strength in young adults with cystic fibrosis. J Clin Endocrinol Metab 99(9):3399-3407

Saha PK, Xu Y, Duan H, Heiner A, Liang GY (2010) Volumetric topological analysis: a novel approach for trabecular bone classification on the continuum between plates and rods. IEEE Trans Med Imaging 29(11):1821-1838

Scanco Medical AG (1997) MicroCT 20 user's guide, Software Revision 2.1, pp 54-55

Stauber M, Muller R (2006) Volumetric spatial decomposition of trabecular bone into rods and plates-a new method for local bone morphometry. Bone 38(4):475-484

Stein EM, Kepley A, Walker M, Nickolas TL, Nishiyama K, Zhou B, Liu XS, McMahon DJ, Zhang CY, Boutroy S, Cosman F, Nieves J, Guo XE, Shane E (2014) Skeletal structure in postmenopausal women with osteopenia and fractures is characterized by abnormal trabecular plates and cortical thinning. J Bone Miner Res 29(5):1101-1109

Tjong W, Kazakia GJ, Burghardt AJ, Majumdar S (2012) The effect of voxel size on high-resolution peripheral computed tomography measurements of trabecular and cortical bone microstructure. Med Phys 39(4):1893-1903

Ulrich D, van Rietbergen B, Lain A, Ruegsegger P (1995) The ability of three-dimensional structural indices to reflect mechanical aspects of trabecular bone. J Bone 25:55-60

Ulrich D, van Rietbergen B, Weinans H, Ruegsegger P (1998) Finite element analysis of trabecular bone structure: a comparison of image-based meshing techniques. J Biomech 31(12):1187-1192

van Rietbergen B, Ito K (2015) A survey of micro-finite element analysis for clinical assessment of bone strength: the first decade. J Biomech 48(5):832-841

Van Rietbergen B, Weinans H, Huiskes R, Odgaard A (1995) A new method to determine trabecular bone elastic properties and loading using micromechanical finite-element models. J Biomech 28:69-81
Vanderoost J, Jaecques SVN, Van der Perre G, Boonen S, D'hooge J, Lauriks W, van Lenthe GH (2011) Fast and accurate specimenspecific simulation of trabecular bone elastic modulus using novel beam-shell finite element models. J Biomech 44(8):1566-1572

Varga P, Dall'Ara E, Pahr DH, Pretterklieber M, Zysset PK (2011) Validation of an HR-pQCT-based homogenized finite element approach using mechanical testing of ultra-distal radius sections. Biomech Model Mechanobiol 10(4):431-444

Vasilic B, Rajapakse CS, Wehrli FW (2009) Classification of trabeculae into three-dimensional rodlike and platelike structures via local inertial anisotropy. Med Phys 36(7):3280-3291

Vilayphiou N, Boutroy S, Sornay-Rendu E, Van Rietbergen B, Munoz F, Delmas PD, Chapurlat R (2010) Finite element analysis performed on radius and tibia HR-pQCT images and fragility fractures at all sites in postmenopausal women. Bone 46(4):1030-1037

Vilayphiou N, Boutroy S, Szulc P, van Rietbergen B, Munoz F, Delmas PD, Chapurlat R (2011) Finite element analysis performed on radius and tibia HR-pQCT images and fragility fractures at all sites in men. J Bone Miner Res 26(5):965-973

Wang J, Zhou B, Liu XS, Fields AJ, Sanyal A, Shi XT, Adams M, Keaveny TM, Guo XE (2015) Trabecular plates and rods determine elastic modulus and yield strength of human trabecular bone. Bone 72:71-80

Wegrzyn J, Roux JP, Arlot ME, Boutroy S, Vilayphiou N, Guyen O, Delmas PD, Chapurlat R, Bouxsein ML (2010) Role of trabecular microarchitecture and its heterogeneity parameters in the mechanical behavior of ex vivo human L-3 vertebrae. J Bone Miner Res 25(11):2324-2331

Zhou B, Wang J, Yu EY, Zhang Z, Nawathe S, Nishiyama KK, Rey Rosete F, Keaveny TM, Shane E, Guo XE (2016) High-resolution peripheral quantitative computed tomography (HR-pQCT) can assess microstructural and biomechanical properties of both human distal radius and tibia: ex vivo computational and experimental validations. Bone 86:58-67

Publisher's Note Springer Nature remains neutral with regard to jurisdictional claims in published maps and institutional affiliations. 